\section{FIB Lift-Out and Milling of Cylindrical Specimens for Electron Tomography (or Atom Probe Field Ion Microscopy)}

\author{
Lucille A. Giannuzzi \\ FEI Company \\ LGiannuzzi@FEICO.com
}

\section{Introduction}

Electron tomography using transmission electron microscopy (TEM) and related techniques (e.g., scanning transmission electron microscopy (STEM) or energy filtered TEM (EFTEM)) allow for 3-D microstructural and elemental mapping of specimens, and has been used successfully in the biological sciences where massthickness contrast dominates these mostly amorphous materials [1]. Z-contrast STEM imaging via high angle annular dark field (HAADF) tomography has also been used successfully in the physical sciences. STEM, EFTEM, and holography tomography are more useful techniques for crystalline materials, since diffraction contrast in conventional TEM images can hinder image reconstruction. Typical tomography routines utilize conventional electron transparent foils, whereby the dimensions of the specimen perpendicular to the electron beam may be orders of magnitude

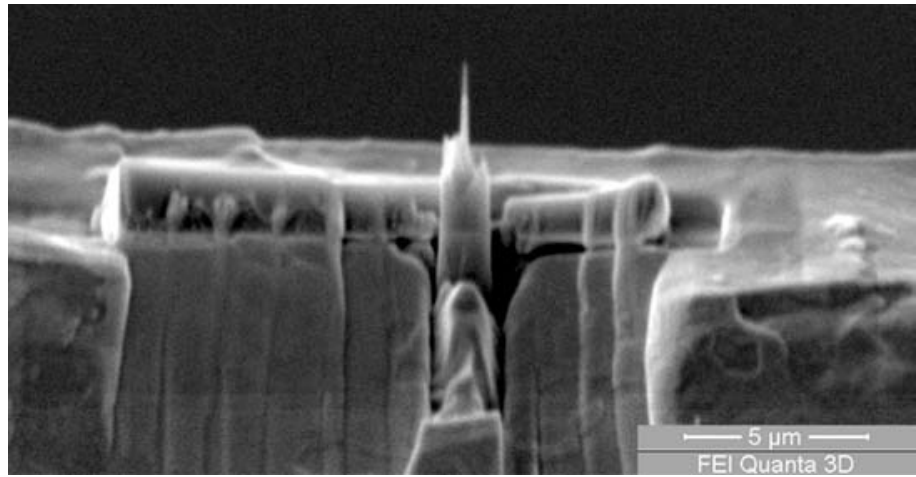

Figure 1. SEM image of in-situ lift-out specimen FIB milled to electron transparency in a cylindrical shape.

greater than the specimen thickness parallel to the electron beam. Using this conventional specimen geometry, the effective specimen thickness increases as the specimen is tilted through the \pm 70 degrees necessary for the tomographic acquisition process. For example, a $100 \mathrm{~nm}$ thick specimen tilted to \pm 70 degrees will have an effective thickness of $292 \mathrm{~nm}$. Thus, performing EFTEM at higher tilts can become impossible since multiple scattering events can hinder the acquisition of adequate or reliable maps. In addition, changes in effective specimen thickness due to specimen tilting can also vary the contrast in HAADF STEM images or alter the phase portion of the image in electron holograms. In order to overcome specimen thickness limitations with tilting, site-specific cylindrical electron transparent focused ion beam (FIB) milled specimens can be prepared using the in-situ lift-out technique [2] in conjunction with previously developed techniques for the preparation of atom probe specimens [3]. The preparation of cylindrical specimens for electron tomography applications was described in an earlier report [4]. Others have also shown that FIB preparing electron transparent rectangular specimens can also be useful for high resolution microscopy as well as for STEM imaging and the acquisition of $\mathrm{x}$-ray maps $[5,6]$.

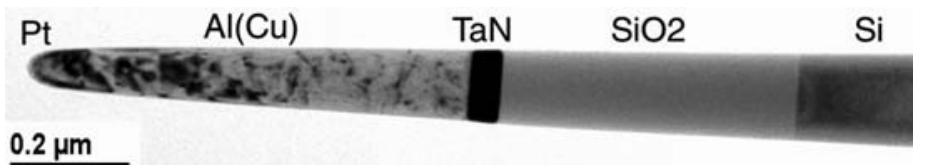

Figure 2. BF TEM image of the specimen shown in figure 1.

\section{Experimental Techniques}

An FEI Quanta 3D DualBeam instrument (FIB + ESEM on the same platform) was used to prepare a cross-section TEM specimen from $\mathrm{Al}(\mathrm{Cu}) / \mathrm{TaN} / \mathrm{SiO}_{2}$ blanket layers on a Si substrate. A large wedge shaped specimen having approximate dimensions $10 \mu \mathrm{m} \mathrm{x}$ $10 \mu \mathrm{m} \times 5 \mu \mathrm{m}$ was fully FIB milled free and then manipulated and secured onto a TEM grid using an Omniprobe Nanomanipulator. A $1 \mu \mathrm{m} \times 1 \mu \mathrm{m} \times 1 \mu \mathrm{m}$ Pt layer was ion beam deposited on the top of the specimen to mark the region of interest. The presence of the Pt layer also served as a monitor of the specimen quality by observing the FIB milling process in real-time using a solid state backscatter electron detector via simultaneous patterning and imaging (i.e., SPI mode). The electron transparent cylindrical specimen was FIB milled using concentric circles where the area to be milled was defined by an inner and outer diameter. Relatively large ion beam currents were used in conjunction with large circle patterns to FIB mill large amounts of material. The beam currents and dimensions of the circle pattern were decreased systematically and monitored via SPI mode until the protective Pt layer was nearly FIB milled away. Figure 1 shows a low magnification secondary electron SEM image of the finished cylindrical TEM specimen prepared using the in-situ lift-out method. The collection of an electron tomography tilt series was then performed with the long axis of the specimen
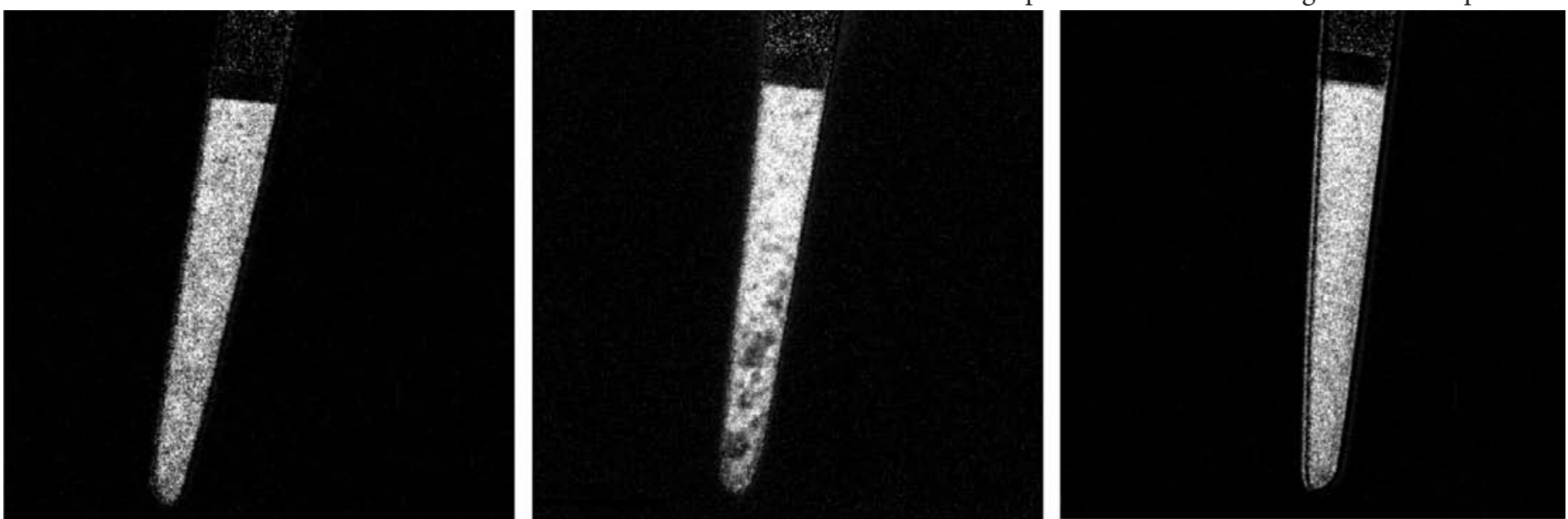

Figure 3. EFTEM Al maps obtained at $-60^{\circ}$ (left), $0^{\circ}$ (center), and $+60^{\circ}$ (right) tilt. 

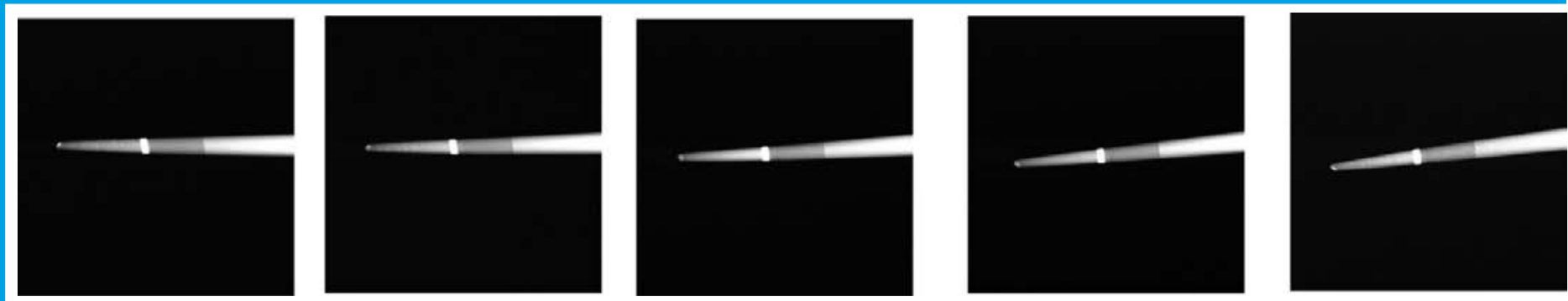

Figure 4. HAADF STEM images of the cylindrical specimen $(-70,-35,0,+35,+65)$

cylinder in the plane of tilting such that the specimen thickness was nearly the same throughout all tilt angles. (S)TEM analysis was performed on an FEI TF30 operating at $300 \mathrm{keV}$.

\section{Results and Discussion}

Figure 2 shows a bright field TEM image of the cylindrical specimen. The individual material layers are labeled. Note that the Pt layer at the tip of the specimen has a radius of $\sim 80 \mathrm{~nm}$. EFTEM Al maps obtained at tilt angles of $-60^{\circ}, 0^{\circ}$, and $+60^{\circ}$ are shown in figure 3. Note that since the tilt axis is along the long axis of the cylindrical specimen, the specimen thickness does not significantly change with tilt, and therefore, EFTEM images may be obtained even at high tilt angles. Figure 4 shows HAADF STEM images obtained at $-70^{\circ},-35^{\circ}, 0^{\circ},+35^{\circ},+65^{\circ}$ tilts. Note that the image contrast for each individual layer is consistent throughout the tilt series since the specimen thickness remains approximately the same as a result of the specimen geometry.

\section{Summary}

A method for preparing cylindrical in-situ lift-out specimens that is suitable for electron tomography techniques has been de- scribed. This specimen geometry is not limited to the examples shown herein, and can also be used to collect images for holography tomography, as well as other TEM tomography techniques not specifically mentioned here. In addition, these techniques may also be used to directly prepare probe tips for atom probe field ion microscopy.

\section{Acknowledgements}

The specimen was obtained courtesy of NanoSpective Inc. TEM tomography was performed with a specimen holder courtesy of Fischione Instruments. Special thanks to C. Kuebel, R. Schoenmakers, and R. Perquin for helpful discussions.

\section{References}

[1] Ziese et al., J. Struct. Biol. 138, (2002), 58.

[2] Giannuzzi et al., in Analysis Techniques of Submicron Defects, 2002 Supplement to the EDFAS Failure Analysis Desktop Reference, ASM Int., Materials Park, Ohio (2002), 29-35.

[3] Martens et al., Microscopy and Microanalysis, 6, suppl. 2, (2000), 522.

[4] Schwarz and Giannuzzi, Micros. Microanal, 10(suppl 2) (2004) 142.

[5] Yaguchi et al., Micros. Microanal, 10(suppl 2) (2004) 1164.

[6] Yaguchi et al., Micros. Microanal. 10(suppl 2) (2004) 1030.

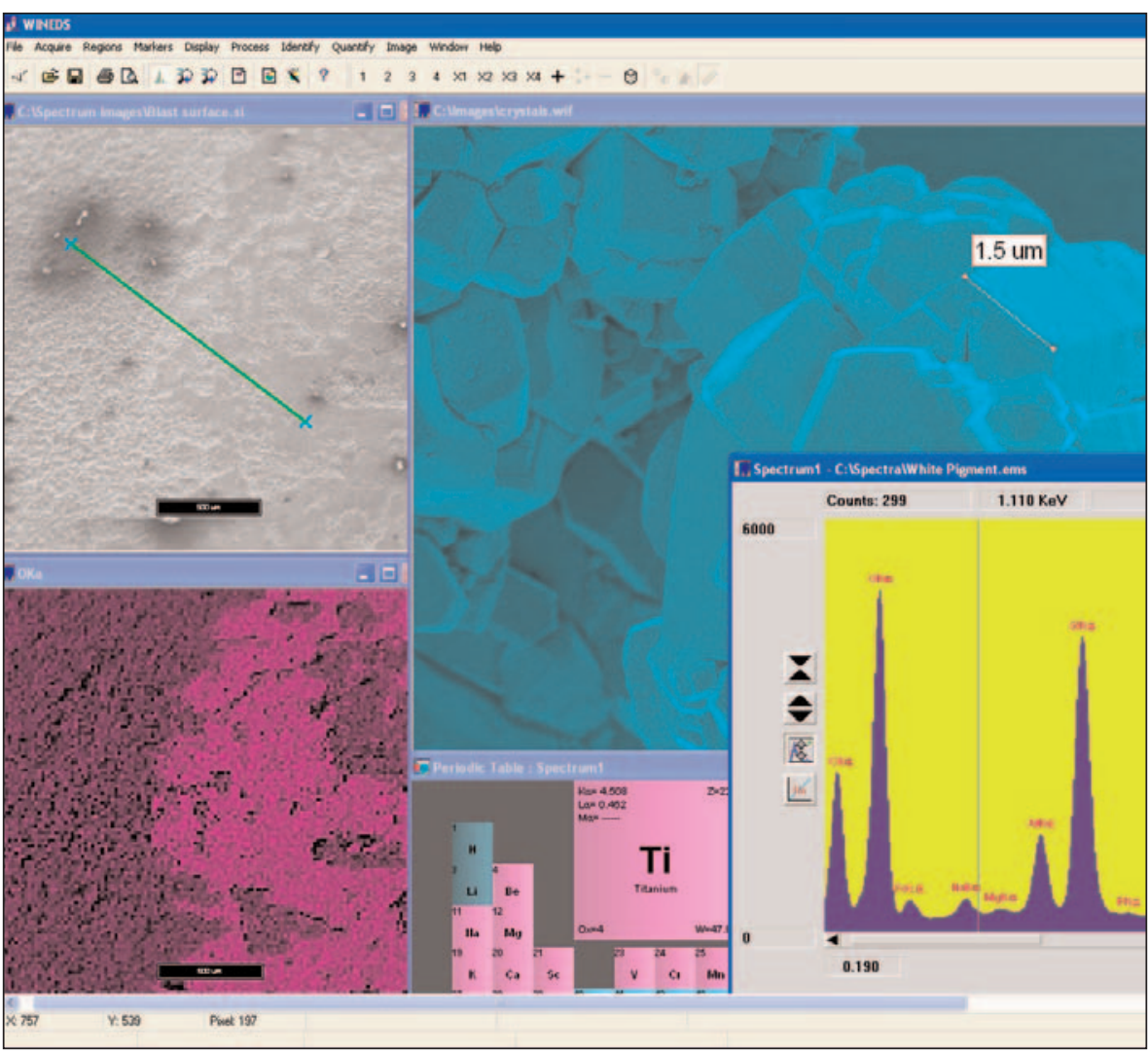

Web + www.tsi.com.au

Email + info@tsi.com.au

216 Drummond St Carlton 3053 Victoria Australia

TEL +61396632738 | FAX +61396633680

\section{UK Agent:}

Cartledge Scientific Instruments

www.c-s-i.demon.co.uk | bill@c-s-i.demon.co.uk Tel/Fax: 1614325355

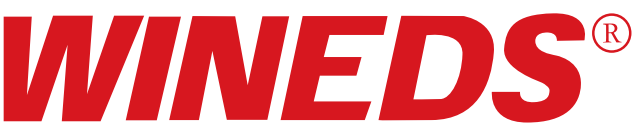

HIGH PERFORMANCE XRAY MICROANALYSIS

INTUITIVE SYSTEM : EASY TO USE, EASY TO GET RESULTS

+ Full Spectrum Imaging and Digital Mapping

+ New generation Digital Pulse Processor

+ Quantitative Analysis

+ Easy report generation

+ Complete range of $\mathrm{X}$-ray detectors

With over 10 years experience in the design of EDX systems for Windows ${ }^{\mathrm{TM}}$, we know how to make microanalysis software intuitive.

In fact, WINEDS behaves just like all your other Windows ${ }^{\mathrm{TM}}$ applications.

So you probably already know how to use it! 